\title{
A COMPARATIVE STUDY ON WAVELET BASED IMAGE DENOISING
}

\author{
Nisha Joy \\ Assistant Professor, T. John Institute of Technology, Department of Electronics and Communication, Bengaluru, \\ Karnataka, India. \\ nishajoy@tjohngroup.com
}

\begin{abstract}
The field of image processing deals with a major issue, i.e., the suppression of noise from the wanted images. The intention of this message is to highlight some of the unique properties of spline wavelets. In this paper image denoising is performed using simulated noise images with various characteristics with the help of semi-orthogonal spline wavelets in comparison with CDF 9/7 wavelets. B-spline analysis can be utilized for different signal/imaging applications such as compression, prediction, and denoising. The exquisite features of wavelet transforms are utilized in the area of image processing which perform better compared to other transforms. Simulated noise images are used to evaluate the denoising performance of $b$-spline wavelets with the help of Bayes Shrink algorithm and along with another wavelet-based denoising like Cohen-Daubechies-Feauveau (CDF 9/7). It is shown through experimental results that, for certain images and input noise levels, the orthogonal b-splines give the best peak signal-to-noise ratio (PSNR), as compared to standard wavelet bases (Daubechies wavelets). Illustrative results that demonstrate the difference in efficiency of the approaches are presented.
\end{abstract}

Keywords: Wavelet Transforms, Spline Wavelets, Image Denoising, Thresholding.

\section{INTRODUCTION}

An image is many a times polluted by noise when it is obtained and as well as during the process of getting it transmitted. The main idea of Image denoising is to trim down the additive noise while keeping intact as much as possible significant features of the image. In the recent years there has been some amount of research on wavelet transforms for signal de-noising because wavelets provides a suitable platform for separating noisy signal from the image signal. The field of image processing uses wavelets because of their varied advantages. Researchers are now in a dilemma of selecting from a variety of bases. While the inclination of the "best" wavelet relies on upon the particular application, it can be helpful to remove various properties and elements that are of general enthusiasm to the client. The guideline aim of this paper is to introduce a rundown of contentions in backing of splines. It has been distinguished to be selective in various ways. The other transforms are way behind the splines in terms of performance. The splines have a precise formula in both the time and frequency domain unlike most other wavelet bases, which very much facilitates their management.

In the next section of the paper, the discussion is related to wavelet based denoising paying attention to multiresolution characteristics and thresholding. It is followed by B-splines and their features. The next section deals with the results proving the plus points of the splines. The paper is winded up with conclusion as the next part. Undeniably, it has been portrayed that the best orthogonal fractional b-splines always perform better than the best standard wavelets (Daubechies).

\section{WAVELET BASED DENOISING}

With the intention of reducing noise from images, wavelet transforms have seen to be an efficient tool. The tree structure of wavelet transforms is one of the properties to be highlighted. Because of its interesting multiresolution property wavelet transform has been a broadly used utensil in image denoising. For the purpose of analyzing a nonstationary signal, it is required to determine its behaviour at a random individual event. Multiresolution analysis gives way to implement this. The Multiresolution analysis is performed by decomposing a signal into a smoothed version of the original signal and a set of detail information at different scales. We can understand this type of decomposition by taking a picture i.e. a $2 \mathrm{D}$ image.

We expel from the photo data that recognizes the most honed edges, leaving another photo that is somewhat obscured. This obscured form of the first picture is a rendering at a somewhat coarser scale. We then recursively rehash the strategy. Every time we get some point of interest data and a more obscured (or smoothed) form of the first picture. Evacuation of the subtle element data compares to a band pass sifting, and era of the smoothed picture relates to a low pass separating. Given the deterioration, we can recreate the first picture.

For Image denoising using wavelets:

a) The DWT of the image is calculated.

b) The wavelet coefficients are subjected to thresholding. The threshold may be universal or sub band adaptive.

c) In order to get the denoised estimate of the image we have to perform IDWT. 


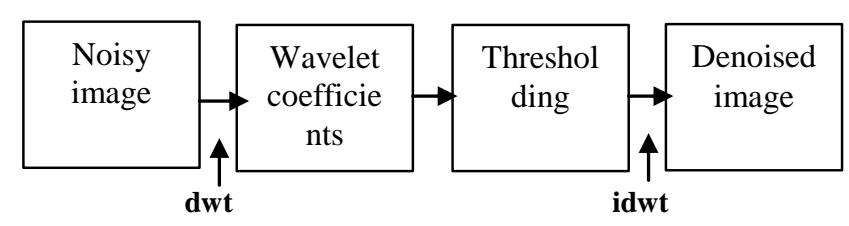

Fig 1. Steps in image denoising

The picture deterioration utilizing wavelet is executed as takes after: In the main level of decay, the picture of concern is part into 4 sub groups, named as the HH, HL, LH and LL sub groups. The askew points of interest of the picture are characterized by the HH sub band; the HL sub band gives the level components while the LH sub band speaks to the vertical structures. The LL sub band is the low determination left over comprising of low recurrence segments and it is this sub band which is further part at more elevated amounts of decay

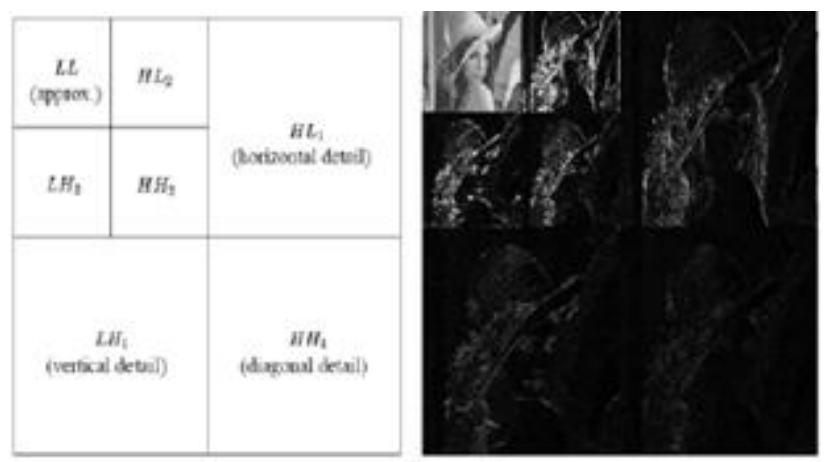

Fig 2. a) 2 decomposition levels b) decomposed image

With the intention of getting visually more pleasing images, soft thresholding methods have been used instead of hard thresholding. $\mathrm{F}(\mathrm{x})=\mathrm{x} \quad$ if $\mathrm{x} \geq \lambda$

$=0 \quad$ otherwise

Alongside keeping up the sign qualities wavelet denoising endeavors to evacuate the commotion present in the sign, regardless of its frequency content. Smoothing is poles apart from wavelet denoising, smoothing removes the high frequency components and retains the lower frequency components. The performance of denoising has different outcomes depending on the wavelet chosen and signal under consideration. Donoho has done most of the work on wavelets which mainly stands on thresholding the DWT of an image.

The technique stands firm on the way that commotion is generally seen as fine-grained structure in the sign, and WT gives a scale-based disintegration. Along these lines, a large portion of the clamor has a tendency to be spoken to by the wavelet coefficients at better scales. On the off chance that we dispose of these coefficients it would bring about a characteristic sifting through of clamor on the premise of scale. Since the coefficients at such scale additionally tend to be the essential transporters of edge data, the technique for Donoho edges the wavelet coefficients to zero if their qualities are beneath a limit. These coefficients are for the most part the ones connected to the clamor. The edge related coefficients of the sign then again, are for the most part over the limit. A discretionary way to deal with hard thresholding is the delicate thresholding, which prompts less serious contortion of the sign of interest. Here the Bayes Shrink strategy has been utilized which is a kind of delicate thresholding for dunking commotion in pictures.

$$
\begin{aligned}
F(x) & =x-\lambda & \text { if } & x \geq \lambda \\
& =0 & \text { if } & x<\lambda \\
& =x+\lambda & \text { if } & x \leq-\lambda
\end{aligned}
$$

Bayes Shrink is a versatile information driven limit for picture denoising by means of wavelet delicate thresholding. The edge has its procedures in a Bayesian casing work and it expect Generalized Gaussian circulation (GGD) for the wavelet coefficient in every point of interest sub band attempting to discover the edge $\mathrm{T}$ which minimizes the Bayesian RiskBayes shrink is an adaptive data driven threshold for image denoising via wavelet soft thresholding. The threshold has its proceedings in a Bayesian frame work and it assumes Generalized Gaussian distribution (GGD) for the wavelet coefficient in each detail sub band trying to find the threshold $\mathrm{T}$ which minimizes the Bayesian Risk

\section{USAGE OF SPLINE WAVELETS}

According to a number of signal processing experts Bsplines have attracted the attention of various engineers and have arrived at scene long time ago. Because of their good points such as differentiability degree and cost/quality trade off matter splines could be portrayed as the next level of trend in the area of wavelets for the purpose of multiresolution analysis in images. We can move from a linear level to a higher-order representation by modifying the spline function. The prominent reason for usage of splines is the theory of wavelet reconstruction. B-slpines are also utilized for reconstruction of signal with the use of polynomial equations. It has been noted that even after viewing the history of splines in the applications related to the field of engineering, they have not been fully studied a image synthesis point of view. They have also been utilized to create wavelets from two sequences which are nonperpendicular. The other uses of splines include construction of shifted b-splines as well as image zooming and interpolation application.

\subsection{Semi-Orthogonal Spline Wavelets}

These kind of wavelets retain their inter-scale orthogonality, but it is not necessary within the same resolution level for the basis functions to be orthogonal to their translates. The very first example of such a case was developed by ChuiWang and Unser-Aldroubi: the wavelet counterparts of $d$ bsplines and the compactly supported spline wavelets. By the inputs of appropriate linear combinations it was observed that we could generate several other semi-orthogonal spline wavelets. These wavelets are adaptable on the grounds that is conceivable to pick the grouping $\mathrm{p}(\mathrm{k})$ in $(14)$ and $\mathrm{w}(\mathrm{k})$ in 
(12) such that the basic capacities have some particular property (e.g. interjection, orthogonality or ideal timerecurrence restriction). We can develop semi-orthogonal splines of any predefined shape with the help of scaling capacities however the double investigation capacity which are splines are typically not. Vast reaction motivation channels are required for the most part. It has been seen that a few of the wavelets and scaling functions in this case can be supported compactly but the dual analysis function which are splines are usually not. Infinite response impulse filters are required generally.

\section{DISCUSSION AND CONCLUSION}

The usage of wavelet transforms are seen in applications of image processing and others like image inpainting and water marking. It is seen in this paper delicate thresholding is utilized for accomplishing better components of edges and smoothness. We have looked at the denoising execution of the b-spline wavelets with $\mathrm{CDF}$ 9/7 biorthogonal wavelets. The point of our correlation is to call attention to the distinctions in term of PSNR which decides every one's productivity because of a specific decision of wavelet bases .The PSNR has been computed using the following PSNR $=10 \log 10\left(255^{2} / \mathrm{MSE}\right)$.

It has been proved by results that the working of the bsplines wavelets can be varied as their parameters can be modified. By making accurate choices of the wavelet parameters these set of wavelets are found to be at the top of other popular wavelets. The following are the illustrative results obtained using MATLAB with SNR of $2 \mathrm{~dB}$.

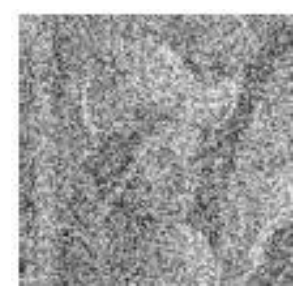

Fig 3. a) Input noisy image spline wavelet

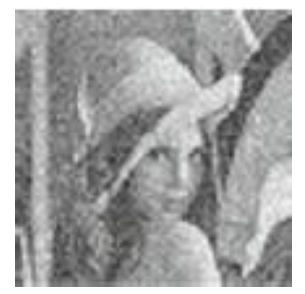

c) Denoised image using CDF 9/7 wavelet

\section{REFERENCES}

[1] Parker, J.I., Keynon, R.V., Troxel, D.E.: Comparison of interpolating methods for image resampling. IEEE Trans. Med. Imaging MI-2, 31-39 (1983)

[2] Keys, R.G.: Cubic convolution interpolation for digital image processing. IEEE Trans. Acoust. Speech Signal Process. 29(6), 1153-1160 (1981)
[3] Hämmerlin,G., Hoffmann, K.-H.:Numerical Mathematics. Undergraduate Texts inMathematics. Springer Verlag, New York (1991)

[4] Schumaker, L.L.: Spline Functions: Basic Theory, Pure and Applied Mathematics. Wiley, New York (1981)

[5] Goswami, J.C., Chan, A.K.: Fundamentals of Wavelets, Theory, Algorithms and Applications. Wiley, New York (1999)

[6] Unser, M.: Splines - a perfect fit for signal and image processing. Signal Process. Mag. 16(6), 22-38 (1999)

[7] Unser, M., Aldroubi, A., Eden, M.: B-Spline signal processing. Part I: theory. IEEE Trans. Signal Process. 41(2), 821-833 (1993)

[8] Unser, M., Aldroubi, A., Eden, M.: B-Spline signal processing. Part II: efficiency design and applications. IEEE Trans. Signal Process. 41(2), 8834-8848 (1993)

[9] Unser, M., Aldroubi, A., Eden, M.: Fast B-spline transforms for continuous image representation and interpolation. IEEE Trans. Pattern Anal. Mach. Intell. 13(3), 277-285 (1991)

[10] Aldroubi, A., Abry, P., Unser, M.: Construction of biorthogonal wavelets starting from any two multiresolutions. IEEE Trans. Signal Process. 46(4), 1130 1133 (1998)

[11] Unser, M., Blu, T.: Fractional splines and wavelets. SIAM Rev. 42(1), 43-67 (2000)

[12] Fahmy, M.F., Elhameed, T.A., Fahmy, G.F.: A fast BSPLINEbasedmethod for image zooming and compression. In: Proceedings of 24th URSI Conference, Ain Shams University, Cairo, Egypt, March 2007 Process. 46(6), 1494-1504 (1998)

[13] b-spline wavelets for signal denoising and image compression $\mathrm{m}$. f. fahmy · gamal fahmy $\cdot$ o. f. fahmy

[14] Which wavelet bases are the best for image denoising ? florian luisiera, thierry blua, brigitte forsterb and michael unsera abiomedical imaging group (big), ecole polytechnique f'ed'erale de lausanne (epfl), lausanne, switzerland bcentre for mathematical sciences, munich university of technology (tum), munich, germany

[15] proc. spie vol. 3169, wavelets applications in signal and image processing v, 1997, pp. 422-431. ten good reasons for using spline wavelets michael unser swiss federal institute of technology (epfl) ch-1015 lausanne, switzerland.

[16] an introduction to wavelets lee a. barford, r. shane fazzio, david r. smith instruments and photonics laboratory hpl-92-124 september, 1992

[17] goswami, j.c., chan, a.k.: fundamentals of wavelets, theory, algorithms and applications. wiley, new york (1999)

[18] Daubechies, Ten lectures on wavelets, Society for Industrial and Applied Mathematics, Philadelphia, PA, 1992.

[19] Aldroubi and M. Unser, "Families of multiresolution and wavelet spaces with optimal properties", Numerical Functional Analysis and Optimization, Vol. 14, No. 5-6, pp. 417-446, 1993.

[20] A Tutorial of the Wavelet Transform Chun-Lin, Liu February 23, 2010

[21] Wavelets and Filter Banks: Theory and Design by Dhaval Shah

[22] Wavelets, Marialuce Graziadei 
[23] Digital Image Processing Using MATLAB, Rafael C. Gonalez, Richard E. Woods, Steven L. Eddins

[24] Biorthogonal Wavelet Transform Digital Image Watermarking, International Journal of Advanced Computer Research (ISSN (print): 2249-7277 ISSN (online): 22777970) Volume-2 Number-3 Issue-5 September-2012

\section{BIOGRAPHIES}

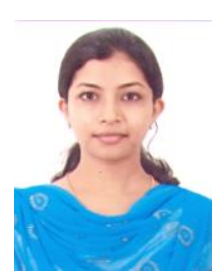

Nisha Joy received M. Tech. Degree with specialization in Digital Communication and Networking from TJIT, Bangalore in 2015. She is currently working as an Assistant Professor in T.John Institute of Technology, Bengaluru, affiliated to the Visveshvaraya Technological University, Belgaum. Her research interests include image compression and image denoising techniques. 